\title{
Crimean Congo Hemorrhagic Fever: Laboratory Findings, Treatment and Prevention
}

\author{
Jamshid Ayatollahi ${ }^{1}$, Seyed Hossein Shahcheraghi ${ }^{1,2 *}$, Razieh Akhondi ${ }^{1}$ and Marzieh Lotfi ${ }^{2}$ \\ ${ }^{1}$ Infectious Diseases Research Center, Shahid Sadoughi University of Medical Sciences, Yazd, Iran \\ ${ }^{2}$ Department of Modern Sciences and Technologies, Faculty of Medicine, Mashhad University of Medical Sciences, Mashhad, Iran
}

${ }^{*}$ Corresponding author: Seyed Hossein Shahcheraghi, Infectious Diseases Research Center, Shahid Sadoughi University of Medical Sciences, Yazd, Iran, Tel: + 98-9132531389; E-mail: shahcheraghih@gmail.com

Received date: June 29, 2017; Accepted date: July 1, 2017; Published date: July 8, 2017

Citation: Ayatollahi J, Shahcheraghi SH, Akhondi R, Lotfi M (2017) Crimean Congo Hemorrhagic Fever: Laboratory Findings, Treatment and Prevention. J Prev Infect Cntrol. Vol.3 No.2:5.

\section{Introduction}

Crimean Congo Hemorrhagic Fever (CCHFV) is a negativesense, single-stranded RNA virus with a 3-segmented genome designated as large (L), medium (M), and small (S), according to size [1]. Its virions are spherical-approximately $100 \mathrm{~nm}$ in diameter-and the lipid envelope is host derived. Recombination events can also occur in short genome regions of virus [2,3]. CCHFV is a potentially fatal tick-borne zoonosis (It is transmitted mainly by tick vector Hyalomma anatolicum, as well as by $H$. marginatum marginatum, $H$. marginatum rufipes, $H$. marginatum turanicum and $H$. marginatum isaaci) characterized by hemorrhage, with a fatality rate of between 10 and 50\% [3]. This disease is widely distributed around the world and has been reported in over 30 countries in Asia, Africa and Europe. Most of the cases were reported from Turkey, Russian, Iran, and Bulgaria [4].

The typical course of $\mathrm{CCHF}$ is a progression through 4 distinct phases, including incubation, prehemorrhagic, hemorrhagic, and convalescence [4]. Healthcare professionals who deal with patients infected with CCHFV are at a high risk of infection due to inadequate biosafety policies and practices and a lack of proper management procedures as a result [4].

Common laboratory findings reveal leukopenia and thrombocytopenia in patients with $\mathrm{CCHF}$ and indicate elevated levels of alanine aminotransferase (ALT), aspartate aminotransferase (AST), creatine kinase (CK) and lactate dehydrogenase (LDH); prolonged prothrombin time (PT); and activated partial thromboplastin time (aPTT). The molecular diagnostic assay recommended for detection of CCHF virus (CCHFV) is reverse transcription polymerase chain reaction (RTPCR) [5].

However, because markets for animal trading are typically located near large cities, CCHFV seroprevalence is also higher among people living in urban areas than people living in rural areas [6].

Until 3 decades ago, there was no appropriate treatment for CCHF. Ribavirin is a nucleoside analogue with a broad spectrum antiviral activity [7]. It has been demonstrated to have an inhibitory effect on the replication of CCHFV in vivo and in vitro, although the efficacy of ribavirin in the treatment of CCHF has not yet been proven. In 2016 a total of 20 patients died in Pakistan from CCHF, the majority being from the Sindh province. However, mortality data since 2012 suggests significant variation in where deaths occur by province by year with no apparent pattern. Most of the cases were reported from Turkey, Russian, Iran, and Bulgaria [6].

It is difficult to prevent or control CCHFV infection in animals and ticks, since the tick-animal-tick cycle continues unnoticed and viral infection in animals is usually not apparent. Additionally, tick vectors are numerous and widespread, making tick control with arachnicide realistic only for well-managed livestock production facilities, which are uncommon in the region [2-5]. The development of vaccines and treatment options are urgent priorities. Vaccine strategies should be based on new recombinant technologies as they are generally safer, better suited for production purposes and probably easier approved by authorities. A better understanding of $\mathrm{CCHF}$ epidemiology is needed for a comprehensive prevention and control programme in the WHO EMR, encompassing human as well as animal and tick populations.

\section{References}

1. Durmuş K, Engin A, Karataş TD, Gözel MG, Altuntas EE (2017) Determination of nasal mucociliary clearance time and nasal symptom in patients with Crimean-Congo hemorrhagic fever. J Med Virol 89: 960-965.

2. Bannazadeh Baghi $\mathrm{H}$ and Aghazadeh $M$ (2016) Include CrimeanCongo haemorrhagic fever virus prevention in pre-travel advice. Travel Med Infect Dis 14: 634-635.

3. Karim AM, Hussain I, Lee JH, Park KS, Lee SH (2017) Surveillance of Crimean-Congo haemorrhagic fever in Pakistan. Lancet Infect Dis 17: 367-368.

4. Ayatollahi J, Shahcheraghi SH, Mirjalili M (2015) Report of nine cases of Crimean-Congo haemorrhagic fever from Iran. Niger Med J 56: 156-159.

5. Atif M, Saqib A, Ikram R, Sarwar MR, Scahill S (2017) The reasons why Pakistan might be at high risk of Crimean Congo haemorrhagic fever epidemic: A scoping review of the literature. Virol J 14: 63 . 
6. Pshenichnaya NY, Leblebicioglu H, Bozkurt I, Sannikova IV, Abuova GN, et al. (2017) Crimean-Congo hemorrhagic fever in pregnancy: A systematic review and case series from Russia, Kazakhstan and Turkey. Int J Infect Dis 58: 58-64.
7. Mozafari O, Shirzadi MR, Shorofi SA, Mozafari A (2016). CrimeanCongo haemorrhagic fever in Persian traditional medicine. Iran J Public Health 45: 1243-1244. 